\title{
Motivation Factors Analysis and Policy Research of Deep Learning
}

\author{
Liyao Bu
}

\author{
School of North China Electric Power University , Baoding 071000, China. \\ 303068677@qq.com
}

Keywords: Deep learning, ISM, AHP, importance degree, motivation factors, policy research.

\begin{abstract}
This article uses Interpretative Structural Modeling Method(ISM) to build deep learning promotion structure diagram of the influence factors, then uses Analytic Hierarchy Process(AHP) to determine the relative importance of various factors, according to the evaluation results it is concluded that the policy guidelines for deep learning to promote the influence degree of the relative maximum conclusion, put forward to promote deep learning better and faster, the government related department should publish relevant policy recommendations, embodied in more research funds, set up special introduction and training of research institutions and researchers, to perfect the theory system. At the same time, the improvement of social awareness will attract more high-tech companies in product research and development, making deep learning applied in more fields.
\end{abstract}

\section{Introduction}

Deep learning has brought a new wave of machine learning, and by the wide attention from academia to industry, also contributed to the coming of the era of "big data + depth model". In the application, deep learning makes voice of intelligent recognition and image understanding, the astonishing progress thereby making big strides to promote artificial intelligence and human-computer interaction. As a result, the great strength of deep learning, and a wide range of promotion has the vital significance.

\section{Hierarchical structure model}

\subsection{Constituent elements of the system.}

Mainly from the internal and external factors to analyze the factors affecting the promotion of deep learning. System is composed of 24 factors, the analysis is as follows.

(1) Improve the algorithm performance.

Compared with shallow learning, deep learning has many advantages, the ability to express complex network objective function aspect, depth institutions neural network can function better characterize the hypervariable shallow structure neural network may not be a good representation of complex high-dimensional functions. However, it is very difficult to train the model, algorithm performance to be further improved.

(2) Enhance computing capacity.

Deep learning multilayer structure is composed of the nonlinear operation unit, a strong need for computing power.

(3) Improve the accuracy rate.

Construction of deep learning is essentially a machine learning model architecture contains multiple hidden layers, large-scale data through training, get a lot more representative feature information, and thus the sample classification and prediction, classification and prediction to improve accuracy. Compared to other conventional algorithms reduce the error rate on a clear advantage.

(4)Fusion with other methods.

Single deep learning methods often can not bring the best results. Fusion is usually a variety of methods or other methods of scoring average, it will bring a higher accuracy rate. Therefore, the depth of learning and integration of other methods, has some significance. 
(5)Improve efficiency.

In general, under the same data set, the larger model, the higher the accuracy of the training, the training speed will be slower. Scale adjustment model, ultra-parameter settings, debugging training and other issues will increase the training time, which seriously affect its efficiency.

(6)Shorten parameter adjustment time.

For any non-linear function, you will be able to find a network of shallow depth and a good enough network to represent the depth of the network only need much less parameters. Set the initial parameters of deep learning very crucial step, it directly affects the quality of the output of the network, so the need for frequent adjustment parameters, thus increasing the training time.

(7)Reduce the computational complexity.

When a network structure with depth $k$ can be compactly express a function, if the depth is less than $\mathrm{k}$ using the network structure of the expression of the function may need to increase the number of exponential scale factor calculation, greatly increased the complexity of the calculations.

(8)Improve stability.

Stability of the algorithm refers to the face of a changing environment to maintain their ability to structure and properties unchanged.

(9)Improve the generalization ability.

Generalization is a machine learning algorithm to sample fresh adaptability. Deep learning needs using the training sample parameter values to calculate the adjustment factor, when a network is limited training sample number is calculated to increase the number factor that generalization ability becomes poor.

(10)Build a unified model.

Now depth model for image and language have similar convolution and down-sampling function modules, a suitable model of how to build a unified deep for different application problems still need to explore.

(11)Improve the robustness.

Robustness is the robustness of the algorithm, that is abnormal and dangerous situation, the structure in order to avoid the collapse of the overall structure of the security objectives. Deep learning needs to be improved in a more advanced algorithms for high dimensional data has more robust aspect.

(12)Use adaptive technology.

Adaptive technology is a decision for the future of technology, which aims to optimize the structure of the algorithm. When the depth model no effective adaptive technology, the distribution of the test data set is different from the distribution of the training set, they are difficult to obtain better performance than conventional models.

(13)Increase data availability.

Increasing access to data is a key driver of the deep learning, machine learning much progress is being driven by data.

(14)Increase the availability of unlabeled data.

At present, the characteristics of a learning mark data is still dominant, but in the real world there are vast amounts of unlabeled data, these data one by adding artificial unmarked label is clearly unrealistic. So, with the development of data collection and storage technologies, we will increasingly focus on the characteristics of a learning unlabeled data to complete the full use of a large amount of unlabeled data.

(15)Use unsupervised learning.

With unsupervised learning algorithms for deep learning of the deep structure layer by layer pre-trained neural network can get a better learning outcomes. Initialize the network not only get a good initial parameter values, and can extract useful information about the input distribution network will help find better global optimal solution. Currently the most successful use of deep learning are supervised learning, unsupervised learning has great potential in the depth of learning.

(16)Policy guidelines. 
At present the relevant departments of the national government has not yet issued a comprehensive policy on the deep learning.

(17)Research Fund.

In 2010, deep learning project is firstly funded from the US Department of Defense DARPA program, participants have the US NEC Research Institute, New York University and Stanford University.

(18)The establishment of research institutions.

In January 2013, at the annual meeting Baidu founder and CEO Robin Li founded Baidu announced a high-profile depth study Institute. This is the first deep learning specialized research institutions.

(19)Introduction and training of research personnel.

Baidu digging deep learning Institute is recruiting talent as Google is also to win over top talent, the current need for more researchers into deep learning.

(20)Improve the theoretical system.

Deep learning algorithm is not yet mature, not formed a complete system of theoretical system needs improvement.

(21)Applications in many fields.

Currently, voice, image and natural language processing is the most widely used deep learning of three areas, including speech recognition and synthesis, machine translation, large-scale image data set, face recognition, pedestrian detection, video classification, behavior recognition. Also have applications in other fields, but has not yet formed a systematic breakthrough. To bring a wider range of applications in academia and industry is the development direction of deep learning.

(22)Improve social concern degree.

In April 2013, "MIT Technology Review" magazine listed the top ten deep learning of the first breakthrough technology in 2013. But awareness among the general public is not very high.

(23)The high-tech company's product development.

Many high-tech companies are exploring a special form of deep learning convolution neural network. IBM and other companies and internationally IFLYTEK, Chinese Academy of Sciences Institute of Automation and other companies or research units have been studied in depth study of speech recognition. Google Android phone's speech recognition system based on neural network is developed, Baidu neural network for a new kind of visual search engine for research and development. Deep learning spread in high-tech fields.

(24)Promote deep learning.

This factor is a problem with the system structural analysis.

\subsection{Construct Interpretative Hierarchical Structural Model.}

By drawing grid figure to analyze binary relation between factors, building up reachable matrix, extracting reduced matrix and hierarchy process, extracting skeleton matrix and drawing multilevel hierarchical directed graph, we get the hierarchical structure of factors to promote deep learning shown in Figure 1. 


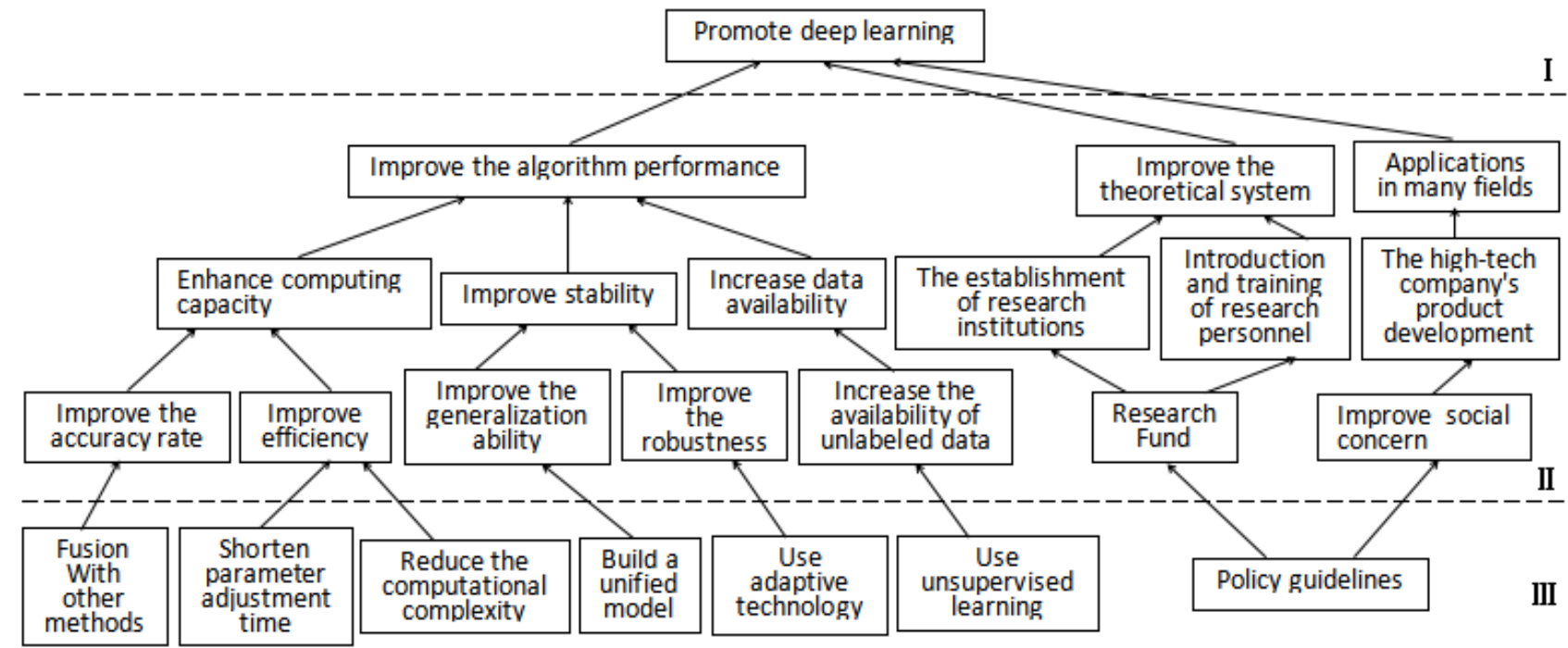

Fig. 1 Hierarchical structure of deep learning motivation factors

\section{Determine the impact of the relative importance of factors}

\subsection{The establishment of evaluation system of hierarchical structure.}

By ISM, we got the hierarchical structure of the evaluation system, shown in Figure 1. It is divided into three levels, namely the target layer (the highest level) I , criteria layer (the intermediate layer) II and program level (the lowest level) III.

\subsection{Calculate the importance degree of the programs.}

After constructing comparison judgment matrix of two factors in all levels, calculating normalized relative importance degree vector with respect to the upper factor and consistency check, using correlation matrix of the overall purpose of the relative importance of the elements of the layers with respect to the system for conversion, and accordingly the program order. The results are shown in Table 1.

Table 1 The importance degree of all programs

\begin{tabular}{cc}
\hline Program & Weight \\
\hline Fusion with other methods & 0.043 \\
Shorten parameter adjustment time & 0.015 \\
Reduce computational complexity & 0.007 \\
Build a unified model & 0.017 \\
Use adaptive technology & 0.017 \\
Use unsupervised learning & 0.065 \\
Policy guidelines & 0.837 \\
\hline
\end{tabular}

\subsection{Determine the relative size of the factors' influence degree.}

By AHP, we got the relative importance of the depth of influence to promote the learning of factors, namely the extent of the relative size of the impact. Policy guidelines $>$ Use unsupervised learning $>$ Fusion with other methods $>$ Build a unified model= Use adaptive technology $>$ Shorten parameter adjustment time> Reduce computational complexity.

\section{Summary}

According to the evaluation results can be seen, the degree of influence on policy guidelines to promote deep learning relative maximum. Depth analysis of the reasons that the self-learning algorithm performance has been good, while the external environment factors has been less complete. Therefore, in order to better promote deep learning faster, national government departments should introduce policies to invest more research funds, the establishment of specialized research and training institutions and the introduction of researchers to improve the theoretical system, while 
increasing the degree of social concern It will attract more high-tech companies in product research and development, so that the depth of learning applied to more areas.

\section{References}

[1]. Yingluo Wang. Systems Engineering. Machinery Industry Press, 2008.

[2]. Baocai Yin, Wentong Wang, Lichun Wang. Review of Deep Learning. Journal of Beijing University of Technology. Vol. 41 (2015) No. 1, p. 48-59.

[3]. Kai Yu, Lei Jia, Yuqiang Chen, et al. Deep Learning: Yesterday, Today, and Tomorrow. Journal of Computer Research and Development. Vol. 50 (2013). No. 9, p. 1799-1804. 